\title{
Protocol for Fit Bodies, Fine Minds: a randomized controlled trial on the affect of exercise and cognitive training on cognitive functioning in older adults
}

\author{
Siobhan T O'Dwyer*1, Nicola W Burton ${ }^{1}$, Nancy A Pachana ${ }^{2}$ and \\ Wendy J Brown ${ }^{1}$
}

\author{
Address: ${ }^{1}$ School of Human Movement Studies, University of Queensland, Australia and ${ }^{2}$ School of Psychology, University of Queensland, \\ Australia \\ Email: Siobhan T O'Dwyer* - sodwyer@hms.uq.edu.au; Nicola W Burton - nburton@hms.uq.edu.au; \\ Nancy A Pachana - npachana@psy.uq.edu.au; Wendy J Brown - wbrown@hms.uq.edu.au \\ * Corresponding author
}

BMC Geriatrics 2007, 7:23 doi:10.1186/147|-23/8-7-23

This article is available from: http://www.biomedcentral.com//47I-23/8/7/23

(c) 2007 O'Dwyer et al; licensee BioMed Central Ltd.

This is an Open Access article distributed under the terms of the Creative Commons Attribution License (http://creativecommons.org/licenses/by/2.0), which permits unrestricted use, distribution, and reproduction in any medium, provided the original work is properly cited.

\begin{abstract}
Background: Declines in cognitive functioning are a normal part of aging that can affect daily functioning and quality of life. This study will examine the impact of an exercise training program, and a combined exercise and cognitive training program, on the cognitive and physical functioning of older adults.
\end{abstract}

Methods/Design: Fit Bodies, Fine Minds is a randomized, controlled trial. Community-dwelling adults, aged between 65 and 75 years, are randomly allocated to one of three groups for 16 weeks. The exercise-only group do three 60-minute exercise sessions per week. The exercise and cognitive training group do two 60-minute exercise sessions and one 60-minute cognitive training session per week. A no-training control group is contacted every 4 weeks. Measures of cognitive functioning, physical fitness and psychological well-being are taken at baseline ( 0 weeks), post-test ( 16 weeks) and 6-month follop ( 40 weeks). Qualitative responses to the program are taken at posttest.

Discussion: With an increasingly aged population, interventions to improve the functioning and quality of life of older adults are particularly important. Exercise training, either alone or in combination with cognitive training, may be an effective means of optimizing cognitive functioning in older adults. This study will add to the growing evidence base on the effectiveness of these interventions.

Trial Registration: Australian Clinical Trials Register: ACTRN0 I2607000 I5 I437

\section{Background}

Cognitive decline is a normal feature of aging. Memory difficulties, slowing mental speed, and decreased mental flexibility become evident in old age, even in older adults without dementia [1-5]. Normal, age-associated cognitive decline is related to declines in everyday functional abilities, and to increases in both formal and informal service use among older adults [6-8]. Interventions which 
improve cognitive functioning are therefore important for the well-being and quality of life of older adults.

\section{Exercise}

Exercise has been suggested as an innovative approach to improving cognitive functioning in older adults. The benefits of exercise for general health and well-being in older adults are already well known [9].

Cross-sectional evidence suggests that older adults who are physically active display better cognitive functioning than their sedentary peers, in areas such as memory, reaction time, and visuospatial skills [10-13]. Prospective and longitudinal findings suggest that physical inactivity is predictive of subsequent cognitive decline and that changes in physical activity patterns over time are associated with changes in cognitive functioning [14-18].

Experimental studies of exercise training programs for older adults have generally shown improvements in cognitive functioning, particularly on measures of information processing speed and executive functioning (e.g. [1921]). While some experimental studies have been less conclusive (e.g. [22-24]), this discrepancy is most likely attributable to methodological differences. These include differences in duration, intensity, and frequency of exercise training, along with differences in control groups, exclusion criteria, and cognitive outcome measures. A meta-analysis by Colcombe and Kramer [25] found that, overall, exercise interventions do have the potential to improve cognitive functioning in older adults, particularly mental speed and executive functioning. The meta-analysis also highlighted that the most beneficial programs are those which have exercise sessions of greater than $30 \mathrm{~min}$ utes duration, include aerobic and strength training components, and target adults aged between 65 and 70 years [25]. As this is a relatively new area of research, additional evidence is required to further support the claim that exercise training can improve cognition.

\section{Linking exercise and cognitive functioning}

Several mechanisms have been suggested to explain the relationship between exercise and cognitive functioning. The main hypothesis is that exercise directly affects the structure and function of the brain. Increases in aerobic capacity (as a result of increases in exercise) are thought to increase cerebral blood flow, improve the transport and utilization of oxygen and glucose in the brain, promote neurogenesis (the creation of new nerve cells), and regulate neurotransmitter synthesis $[14,15,17,18,26-28]$. Some studies have found an association between improvements in aerobic capacity and improvements in cognitive function $[19,21]$. Other studies, however, have found no relationship [22-24,29]. More evidence is required to validate the idea of increased aerobic capacity as a prerequisite for improved cognitive functioning.

Psychological factors may play a role in mediating the relationship between exercise and cognition [30]. Exercise is known to improve psychological well-being [31], and psychological well-being has been associated with cognitive functioning $[32,33]$. A few studies of exercise training and cognitive training have included psychological measures (e.g. [22,34-38]), but results have been mixed. More research is required to elucidate the mediating role of psychological factors in the exercise-cognition relationship [30].

\section{Combination training}

Some researchers have suggested that the benefits of exercise may be further enhanced by combining exercise training with cognitive training [36].

Cognitive training has been the traditional approach to improving cognitive functioning in older adults. It consists of learning and practicing skills and techniques to manage cognitively demanding situations (e.g. using mnemonics to aid recall). While these programs have been successful in improving the specific cognitive function targeted by the training (i.e. memory programs improve memory performance) [39-41], they do not have the potential to provide the physical and cognitive benefits offered by exercise training. A combination of exercise training and cognitive training, however, may provide the best of both worlds.

Fabre and colleagues [36] conducted an experimental study of combination training with four groups: aerobic training, cognitive training, aerobic and cognitive training, and control. They found that all three training groups improved significantly, with the combination training group (aerobic and cognitive training) improving significantly more than the other groups. These findings are limited, however, by the fact that the combination training group appear to have received a greater total number of training sessions per week than the other groups. Given that engaged lifestyles have been linked to cognitive performance in older adults [42-45], it is possible that the results of the combination training group could be a consequence of increased social interaction, rather than an added benefit of cognitive training. The potential of combined exercise and cognitive training could be better investigated by matching the overall training exposure of participants in a dual group to that of participants in an exercise training group.

\section{Feasibility}

Little is known about older adults' perceptions of exercise training programs and combined exercise and cognitive 
training programs. While adherence and compliance data can provide some insight into the feasibility of a training program, qualitative feedback from participants provides information about satisfaction and acceptability. Collection of this type of information also provides an opportunity to identify specific strengths and weaknesses of a program, and to assess the real-life benefits of the training which cannot be measured with clinical tests or surveys $[46,47]$.

\section{Aim}

The aims of the present study are to:

(1) examine the impact of an exercise training program for older adults on cognitive and physical functioning,

(2) compare the impact of exercise training and a combined program of cognitive and exercise training for older adults (which has an equal number of total training sessions per week) on cognitive and physical functioning,

(3) examine associations between changes in aerobic capacity (as a result of exercise) and changes in cognitive functioning;

(4) examine associations between changes in psychological wellbeing (as a result of exercise) and changes in cognitive functioning; and

(5) identify older adults' perceptions of exercise training and combined exercise and cognitive training.

An exercise training program and a cognitive training program have been developed by the first author (SO), and will be evaluated in a randomized controlled trial. The content of these programs is based on previously successful training programs, and current theories regarding the relationships between exercise training, cognitive training, and cognitive functioning.

\section{Methods/Design \\ Study design}

Fit Bodies, Fine Minds is a 16-week, randomized controlled trial of exercise training and combined exercise and cognitive training for older adults. Participants are allocated to one of three groups: exercise-only training; exercise and cognitive training; no-training control. Assessments are conducted at baseline, post-test (16-weeks) and 6-month follow-up (40 weeks). The design is presented in Figure 1. The protocol was approved by the University of Queensland Medical Research Ethics Committee (Approval Number: HMS06/2303)

\section{Setting}

The program is conducted at Riverside Fitness, a privatelyowned gym in Brisbane, Australia.

\section{Study population}

The program is designed for community-dwelling older adults without cognitive impairment. Participants are recruited through healthy aging seminars at local churches, public radio broadcasts, and the 50+ Registry (a registry of research volunteers aged over 50 years, coordinated by the Australasian Centre on Ageing at the University of Queensland). All interested people are provided with a Participant Information Sheet detailing the nature of the program.

Individuals are initially considered eligible if they are

(1) aged between 65 and 75 years,

(2) doing less than 60 minutes of moderate-intensity exercise per week,

(3) living within $10 \mathrm{~km}$ of Riverside Fitness and the University of Queensland; and

(4) available to participate in the program during business hours.

\section{Screening}

Individuals who meet the initial eligibility criteria take part in a telephone interview to screen for cognitive and health problems. People who score below 21 on the Telephone Interview of Cognitive Status (TICS) [48] are excluded from participating in the program. Individuals who report more than one serious health complaint (e.g. recent heart attack, uncontrolled diabetes, or uncontrolled hypertension) are required to obtain written permission from their general practitioner (GP) to take part in the program. Those unable to obtain GP permission are excluded from participating in the program. Individuals who report diagnoses of Alzheimer's Disease, dementia, or recent head injuries are also excluded from the program.

\section{Informed consent}

Participants provide signed informed consent prior to baseline testing.

\section{Sample size}

Ninety-nine participants will be recruited for the study. A sample size of 12 participants in each of the three groups would enable detection of a within-group difference, from baseline to post-test, of 1 item $(\underline{\mathrm{SD}}=1.1)$ on Digit Span and 184 milliseconds $(\underline{\mathrm{SD}}=198)$ on Choice Reaction Time. Twenty participants in each group would enable 


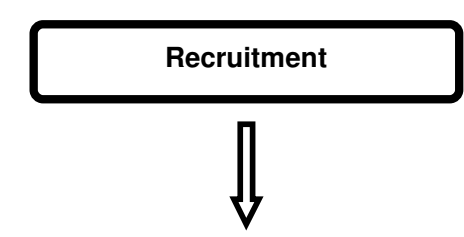

Eligibility Check

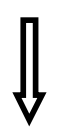

Screening \& GP Approval

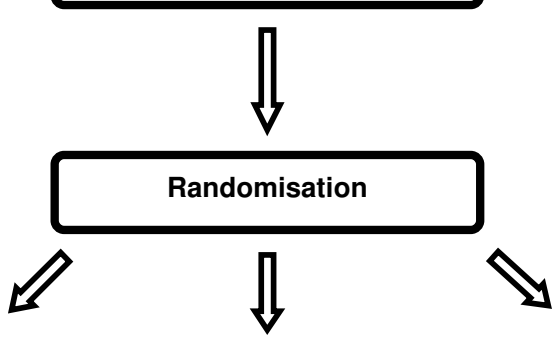

Exercise Only

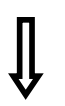

Exercise \& Cognition

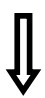

\section{Control}

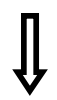

Baseline Assessment (Week 0)

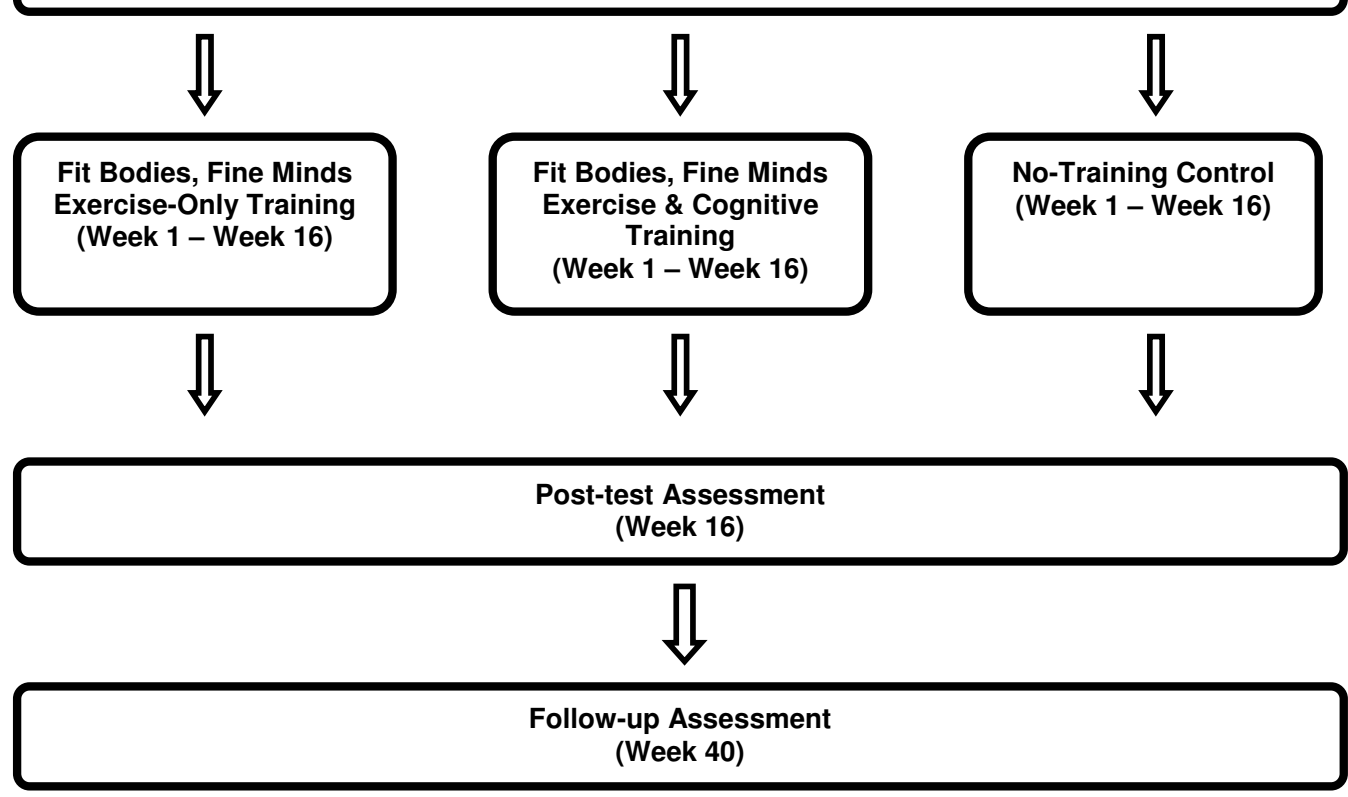

Figure I

The design of the Fit Bodies, Fine Minds study. 
detection of a between-group difference, at post-test, of 2 items $(\underline{\mathrm{SD}}=2.2)$ on Digit Span. Twenty-nine participants in each group would enable detection of a between-group difference, at post-test, of 118.8 milliseconds $(\underline{S D}=160)$ on Choice Reaction Time. These sample size calculations assume a two-tailed alpha level of 0.05 and a power of $80 \%$. The effect sizes and standard deviations are based on results from previous studies of physical activity for cognitive functioning in older adults (e.g. $[11,49])$. To account for attrition over time, the required sample size (29) is increased by $10 \%$ to 33 participants per group.

\section{Randomization}

Participants are randomly allocated using block randomization [50]. Each block contains one exercise-only group allocation, one combined exercise and cognitive training group allocation, and one control group allocation. The order of the groups within each block is randomly generated by selecting papers from a box. The box contains three papers marked Exercise Only, Exercise \& Cognition, and Control, respectively. This process is repeated to generate a list of 25 blocks. Each time a participant meets the eligibility criteria and screening requirements, they are allocated to the next available place on the list.

\section{Blinding}

For logistical reasons, participants, researchers, and instructors are not blind to the allocation of participants.

\section{Intervention}

\section{Exercise-only training}

Participants in this group receive three gym-based sessions per week for 16 weeks. Each session lasts for 60 minutes and includes progressive aerobic and strength training. Each session includes a maximum of 10 participants and is supervised by at least one qualified fitness instructor.

The progression of the aerobic training is adapted from the ACSM Guidelines for Exercise Testing and Prescription [51]. Intensity is prescribed using percentage of Heart Rate Reserve (HRR). Participants on medications which attenuate heart rate (e.g. beta-blockers) are prescribed exercise intensity using Rate of Perceived Exertion (RPE; [52]). The relative progressions of intensity and duration can be seen in Table 1. Intensity is monitored with Polar A3 heat rate monitors. Participants complete the aerobic training on treadmills, stationary bicycles, cross-trainers, and stationary rowing machines.

Strength is assessed, and intensity prescribed, for each participant in the first gym session. Progress is monitored and prescribed by the fitness instructor. The strength training includes exercises to target major muscle groups in the upper body, lower body, and core. The upper and lower body exercises use free weights and machines. The core exercises use a swiss-ball.

Participants stretch major muscle groups at the end of each session.

\section{Exercise and cognitive training}

Participants in this group receive two gym-based exercise sessions and one cognitive training session per week for 16 weeks. Each session lasts for 60 minutes. The exercise training (including the progression of duration and intensity) is identical to that received in the Exercise-Only Group. The cognitive training program includes three modules, covering memory, executive functioning, and mental speed. Each session follows the same format:

\section{(1) theoretical overview}

(2) outline of 'everyday' applications

(3) practice activities

Introductory and revision sessions are conducted at the beginning and end of the program. Table 2 outlines the content of each session. A graduate psychology student leads these sessions in a quiet room away from the main gym area.

\section{Control group}

Participants in this group receive no formal intervention. They are contacted by telephone at Week 4 and 12 to check continued completion of the weekly diary. At Week 8 each participant is posted a cinema voucher and a letter to thank them for their ongoing involvement.

Table I: Duration and intensity progression of aerobic training

\begin{tabular}{cccc}
\hline Week & Duration (min) & Intensity (\% HRR) & Intensity (RPE) \\
\hline 1 & 20 & $50-60$ & $9-11$ \\
2 & 20 & $50-60$ & $9-11$ \\
$3-5$ & 25 & $60-70$ & 11 \\
$6-8$ & 30 & $60-70$ & 11 \\
$9-11$ & 30 & $70-80$ & $11-13$ \\
$12-14$ & 35 & $70-80$ & $11-13$ \\
$15 \& 16$ & 40 & $75-85$ & $13-15$ \\
\hline
\end{tabular}


Table 2: Cognitive training program outline

\begin{tabular}{ccl}
\hline Module & Session & Content \\
\hline Memory & 1 & Aging, Cognitive Functioning, Program Overview \\
& 2 & Introduction to Memory \\
& 3 & Chunking, Organisation, Visualisation \\
Executive Function & 4 & Storytelling, Method of Loci, Mnemonics \\
& 5 & Keywords, Association \\
& 6 & Introduction to Reasoning \\
& 7 & Pattern Identification \\
Information Processing & 8 & Concept Formation, Verbal Fluency \\
& 9 & Attention, Mental Flexibility \\
& 10 & Introduction to Speed of Information Processing \\
& 11 & Simple Speed \\
& 12 & Choice Response Speed \\
& 13 & Memory \\
& 14 & Executive Function \\
& 15 & Speed of Information Processing \\
\hline
\end{tabular}

\section{Adherence and compliance}

Attendance at each cognitive training session is recorded by the instructor. At each exercise training session, participants are provided with individual diaries in which to record the date and the intensity and duration of each activity undertaken in that session. All participants (including control participants) are provided with a weekly diary in which to record physical, social and cognitive activities undertaken outside the program.

\section{Assessment protocol}

Assessment stages

In addition to screening, assessments are conducted at baseline ( 0 weeks), 16-weeks (post-test), and 40 weeks (6month follow-up).

\section{Modes of assessment}

Screening is conducted by telephone. Cognitive and physical assessments are conducted face-to-face at the University of Queensland. Cognitive assessments are conducted by SO. Each cognitive assessment session lasts for 90 minutes. Physical assessments are conducted by an exercise physiologist and last for 30 minutes. Physical assessments are conducted immediately after the cognitive assessments. Self-administered questionnaires are used to assess psychological wellbeing. These are provided after the cognitive assessment, with participants instructed to complete them at home and mail them back within seven days using a provided reply-paid envelope. Participant perceptions of the two training programs are collected using a feedback form included with the other self-report instru- ments. Only training participants complete this feedback form.

\section{Measurements}

The primary outcome measures are indicators of cognitive functioning, aerobic capacity and strength. Secondary outcome measures are anthropometric measurements, resting heart-rate, blood pressure, indicators of psychological well-being and participant perceptions. All measures are assessed at baseline, post-test, and 6-month follow-up, except for the qualitative feedback which is assessed only at post-test.

\section{Screening}

\section{Demographics}

Includes gender, age, education, occupation, nationality, marital status, and language.

\section{Health information}

Includes height, weight, current level of physical activity, hearing, vision, chronic diseases, illnesses, and injuries.

Telephone Interview of Cognitive Status (TICS-m)

This is a brief telephone-administered dementia screening measure, modified slightly from the original to be more suitable for telephone use $[48,53]$. Participants complete a series of short cognitive tasks, such as counting backwards, remembering a list of 10 words, and answering general knowledge questions. Performance on the original TICS has been shown to be highly correlated with performance on the Mini-Mental State Examination $[48,54]$. 
The TICS- $m$ is sensitive and specific [53]. A score of less than 30 (out of a possible 50) on the TICS-m is the cut-off for cognitive impairment [53].

\section{Primary outcome measures: Cognitive functioning}

Three domains of cognitive functioning are assessed: memory, executive functioning, and speed.

\section{California Verbal Learning Test (CVLT-II)}

This assesses the ability to use semantic categories to aid recall (memory) [56]. Participants are presented with words from four semantic categories (e.g. fruit, tools, clothing, herbs), listed in random order. Participants must then recall these words in a number of conditions, including immediate free recall (in which participants are asked to recall as many words as they can, in any order), delayed free recall (approximately 20 minutes after the original presentation), and cued recall (in which participants are asked to recall as many words as they can which fit in each of the categories) [57]. CLVT has satisfactory test-retest reliability [57]. Norms are also available for the CVLT, which has been shown to be somewhat sensitive to the effects of aging and neuropsychological impairment [57]. Scores include the number of words correctly recalled, the number of intrusions, and the number of repetitions. To limit test-retest effects, the original form is used at baseline and 6-month follow-up, with the alternate form used at the post-test assessment (16 weeks).

\section{Logical Memory}

This task is from the Wechsler Memory Scale (WMS-III) [58]. It is a measure of semantic memory. Participants are presented with two brief stories and must recall information from these stories immediately and after a delay of between 10 and 20 minutes. Logical Memory has good test-retest reliability, is not susceptible to practice effects, and norms are available [57]. Logical Memory has been shown to be sensitive to the effects of aging and neuropsychological impairment [57]. Scores include the number of themes recalled and the amount of specific content information recalled [57].

\section{Digit Span}

This is a task from the Wechsler Memory Scale (WMS-III) [58]. It is a measure of working memory. Participants are presented with a number sequence and must immediately recall the numbers. The task progresses in difficulty as number sequences increase in length (minimum $=2$ digits, maximum $=9$ digits). Digit Span has two components. In the first part participants must recall the number sequences in the order presented (Digits Forward). In the second part participants must recall the number sequences in the reverse order to which they were presented (Digits Backward). Digit Span has good test-retest reliability, is not susceptible to practice effects and norms are available. Scores include the number of correctly recalled digits forward, the number of correctly recalled digits backward, and the total number of correctly recalled digits.

\section{California Older Adult Stroop Test (COAST)}

Stroop Tests are a common measure of executive functioning. COAST has been specially developed for use with geriatric populations [59]. It has three components. For the first task participants must identify the names of colours. For the second task participants must read colour name words. In the final task participants must identify the colour in which each colour name word is printed (e.g. the word RED is printed in green ink, so the correct participant response is "Green"). Stroop Tests generally have shown good test-retest reliability and only modest practice effects [57]. Stroop Tests have also been shown to be sensitive to the effects of aging and neuropsychological impairment, and some norms are available [57]. Scores include completion time, number of errors and number of self-corrected errors.

\section{Sorting Test}

This task is from the Delis-Kaplan Executive Function Scale (DKEF-S) [60]. Participants are presented with a set of six cards. The cards have words printed on them, and vary in colour and size. There are up to eight ways (rules), according to which the cards may be sorted into groups of three. In the Free Sorting condition, participants must sort the cards in as many different ways as possible and identify the rule by which they have sorted. The Sorting Test has been shown to be sensitive to the effects of aging and neuropsychological impairment [57]. The Sorting Test has good test-retest reliability and norms are available [60]. Scores include the number of correct sorts and the number of correct rule descriptions.

\section{Matrix Reasoning}

This is a task from the Wechsler Adult Intelligence Scale (WAIS) [61]. It is a measure of executive functioning. Participants are presented with a visual pattern that has one part missing. Participants must select, from multiple choice options, the picture which completes the pattern. The patterns progress in difficulty. Matrix Reasoning has good test-retest reliability and norms are available [61]. The score is the number of patterns correctly solved.

\section{Symbol Digit Modalities Test (SDMT)}

This is a measure of psychomotor speed [62]. It is a written task requiring participants to substitute numbers for symbols according to a key. Participants must complete as many substitutions as possible in 90 seconds. The SDMT has good test-retest reliability, is not susceptible to practice effects and is sensitive to the effects of aging and neu- 
ropsychological impairment. Norms are available [57]. The score is the number of correct substitutions.

Timed Instrumental Activities of Daily Living (TIADL)

This is a measure of applied information processing speed [63]. Participants complete five common tasks of daily living, according to set instructions. These tasks cover communication, money, food preparation, shopping, and medications, and incorporate actual everyday objects [63]. The score is based on the time taken to complete each task, with an adjustment for any errors.

\section{Simple reaction time}

Simple Reaction Time tasks are frequently used to measure psychomotor speed [64]. Computer-based, these tests require participants to press a button when a stimulus (e.g. a light or object) appears on the screen. Marked slowing of reaction time is evident with age and neurological impairment. Consistent with current practice [64], a Simple Reaction Time Task was developed specifically for this project. The Simple Reaction Time Task is run on a Toshiba Tecra A2 Laptop. For this task, 4 taps (faucets) are presented horizontally (and equidistant) across the screen. Participants are required to press the Space Bar when water starts to drip from one of the taps. A warning tone and message ("Get Ready") appear at the top of the screen before each trial. To avoid anticipatory responses, the interval between the warning and stimulus onset is variable. Participants complete 50 trials, with response time recorded in milliseconds from stimulus onset. Responses less than 100 millseconds are considered invalid. Scores are mean reaction time and standard deviation of reaction time.

\section{Choice reaction time}

Choice Reaction Time Tasks assess speed and concentration [64]. In these tests participants are presented with one stimulus and multiple response options. Responses are usually more delayed than in Simple Reaction Time Tasks, because participants must make a decision before responding [64]. The Choice Reaction Time Task was also developed specifically for this project. The Choice Reaction Time Task is run on a Toshiba Tecra A2 Laptop. For this task, 4 taps (faucets) are presented horizontally (and equidistant) across the screen. The keys D, F, J and K correspond to each tap in order from left to right. Participants are instructed that when water starts to drip from one of the taps, they are to press the corresponding key. A warning tone and a message ("Get Ready") at the top of the screen precede each trial. To avoid anticipatory responses, the interval between the warning and stimulus onset is variable. Participants complete 50 trials, with response time recorded in milliseconds from stimulus onset. Any errors are also recorded. Responses less than 100 millseconds are considered invalid. Scores are mean reaction time, standard deviation of reaction time, and number of errors.

\section{Primary outcome measures: aerobic capacity and strength Six-Minute Walk Test (SMWT)}

This is adapted from the Senior Fitness Test [65]. It is a functional measure of aerobic endurance. A rectangular course, measuring $3 \mathrm{~m} \times 15 \mathrm{~m}$, is marked out for participants to walk around. Two chairs are placed inside the course for participants who need to rest during the test. The score is the number of metres walked in six minutes. The number of rests taken or adaptations used (e.g. walking sticks etc) is noted with the score.

\section{Modified Astrand-Ryhming Cycle Ergometer Test}

This is a multi-stage, sub-maximal exercise test, modified from the Astrand-Ryhming protocol [51]. It is conducted on a cycle-ergometer and is a measure of aerobic fitness. This test is similar to the Astrand-Ryhming protocol, except that work-rate is adjusted after the second minute, according to heart-rate and age (see Table 3 ). The initial power level is set at $0.5 \mathrm{kp}$ for sedentary older women, and $1.0 \mathrm{kp}$ for sedentary older men. This modified protocol is regularly used with clinical populations in research conducted at the School of Human Movement Studies, University of Queensland. The Astrand-Ryhming nomogram (including the age correction factor) is used to estimate maximal oxygen uptake $\left(\mathrm{VO}_{2} \mathrm{max}\right)$ [51].

\section{0-Second Chair Stand}

This is taken directly from the Senior Fitness Test [65]. It is a functional measure of lower body strength. Participants are seated in a chair, with feet flat on the floor. The

Table 3: Power alterations according to age and heart rate after $2^{\text {nd }}$ minute (beats per minute)

\begin{tabular}{lccc}
\hline & Heart rate 65 years old & Heart rate 70 years old & Heart rate 75 years old \\
\hline Raise power level by & & & $<90$ \\
$1.0-1.5 \mathrm{kp}$ & $<92.5$ & $90-109$ & $87.5-106.5$ \\
$0.5-1.0 \mathrm{kp}$ & $92.5-111.5$ & $110-119$ & $107.5-116.5$ \\
$\leq 0.5$ & $112.5-121.5$ & $>140$ & $>137.5$ \\
Lower power level by & $>142.5$ & $130-139$ & $127.5-136.5$ \\
$0.5-1.0 \mathrm{kp}$ & $132.5-141.5$ & & \\
$\leq 0.5 \mathrm{kp}$ & & & \\
\hline
\end{tabular}


score is the number of full stands completed in 30 seconds, with arms folded across the chest. Any use of adaptations (e.g. pushing off the legs, using the arms of the chair) is noted with the score.

\section{Arm Curl}

This is taken directly from the Senior Fitness Test [65]. It is a functional measure of upper body strength. Participants are seated in a chair and given a small dumbbell ( 2 $\mathrm{kg}$ for women, $4 \mathrm{~kg}$ for men). The score is the number of bicep curls completed in 30 seconds. Any adaptations (e.g. use of a lighter weight) are noted with the score.

\section{Secondary outcome measures: Anthropometrics and physiology}

Anthropometrics

Measures of height $(\mathrm{cm})$, weight $(\mathrm{kg})$, hip girth $(\mathrm{cm})$ and waist girth $(\mathrm{cm})$ are taken using standard protocols [51].

\section{Resting heart rate}

Resting heart rate is measured by palpation of the radial artery while participants are seated. The number of beats recorded in 10 seconds is multiplied by 6 , to give beats per minute.

\section{Blood pressure}

Diastolic and systolic blood pressure $(\mathrm{mm} \mathrm{Hg})$ are measured using standard protocols [51].

\section{Secondary outcome measures: Psychological well-being Memory Complaint Questionnaire (MAC-Q)}

This is a six-item scale of self-reported memory decline [66]. Participants rate current memory ability against past performance for given situation (e.g. remembering the name of a person just introduced to you), on a 5-point scale from much better now to much worse now. The scale reliability is .57, with test-retest reliability of .67 [66]. Scores are summed, with double weighting for the final item (a comparison of general memory abilities). A higher score indicates greater self-reported decline.

\section{SF-36: Mental Health}

The Mental Health component score from the Medical Outcomes Study Short Form (SF-36; [67] reflects emotional wellbeing as measured on four subscales: vitality (items on energy and fatigue), social functioning (items on the impact of physical and emotional health on social activities), role-emotional (items on the impact of emotional problems on everyday activities), and mental health (items on happiness, nervousness and depression). The SF-36 is a widely used measure of wellbeing and Australian norms are available. A scoring algorithm is provided in the manual [68].

\section{Goldberg Anxiety and Depression Scale (GADS)}

This is an 18 -item measure of symptoms of anxiety and depression, derived from psychiatric interview protocols [69]. Participants answer Yes or No to questions about their feelings in the past month, such as have you been irritable? and have you felt hopeless? The scale has been validated against clinical diagnoses of anxiety and depression, and found to have good specificity and sensitivity [69]. Higher scores reflect increased symptoms of anxiety and depression.

\section{Health-Related Hardiness Scale (HRHS)}

This scale assesses health-related hardiness, a "personality construct that moderates the stress-illness relationship" [70]. The construct is comprised of three dimensions: control, commitment, and challenge. The control dimension is associated with a sense of control over one's health and confidence in one's skills for managing health. The Control subscale of the HRHS is used in this study. Participants respond to items such as I can avoid illness if I take care of $m y$ self, on a 6-point scale from strongly disagree to strongly agree. The Control sub-scale reliability is .91, with test-retest reliability of .76 [70]. Higher scores reflect an increased sense of control over health.

\section{Secondary outcome measures: Participant perceptions of the program}

Qualitative responses about the training programs are gathered from participants to assess the suitability of the programs for older adults.

\section{Program feedback form}

This includes specific questions inviting participants to appraise the Fit Bodies, Fine Minds program. Written responses are invited on issues such as positive and negative outcomes of participation in the program, barriers to and facilitators of participation, enjoyment of activities and setting, and suitability of duration and frequency of sessions.

The form also includes two questions with 5-point Likertscale response options. The first question is How enjoyable was Fit Bodies, Fine Minds? The response options are extremely enjoyable, very enjoyable, quite enjoyable, somewhat enjoyable, and not at all enjoyable. The second question is How did you feel about attending Fit Bodies, Fine Minds? The response options are I looked forward to it ALL of the time, I looked forward to it MOST of the time, I looked forward to it SOME of the time, I could take it or leave it, and I DID NOT look forward to it at all.

\section{Analyses}

Analysis of variance techniques will be used to assess changes over time (baseline, post-test, follow-up), and differences between groups, on the measures of aerobic 
capacity and cognitive functioning. Mean scores and variability scores will be used in these analyses. Variability scores may provide a more sensitive indicator than mean scores, of changes in cognitive functioning in older adults [71]. Where appropriate age, education and gender will be included as covariates. The Reliable Change Index will be used to determine the percentage of participants who demonstrate a significant change in performance, relative to their individual baseline $[72,73]$. This approach may provide a more accurate picture of changes experienced by participants than simple group differences $[72,73]$. Regression techniques will be used to identify the influence of changes in aerobic capacity on cognitive functioning. Regressions techniques will also be used to identify the influence of changes in psychological wellbeing on cognitive functioning.

The qualitative data will be analysed to identify themes related to participant perceptions of the program.

\section{Discussion}

"Nothing could be of greater importance to the imperatives imposed by global aging" than research into the benefits of exercise for cognition, according to Bortz [74]. This randomized controlled trial is designed to examine the effects of exercise training and combined exercise and cognitive training on the cognitive and physical functioning of community-dwelling older adults. It is hypothesized that

(1) both training groups will show significant improvements on measures of cognitive and physical functioning, relative to the control group,

(2) the combined training group (exercise and cognitive training) will show greater cognitive improvements than the exercise-only training group,

(3) improvements in cognitive functioning will be associated with improvements in aerobic capacity,

(4) improvements in cognitive functioning will be associated with improvements in psychological well-being; and

(5) participants will report positive experiences and outcomes from the two training programs.

If the training programs detailed in this protocol provide cognitive and physical benefits and are acceptable to older adults, they may represent a viable approach to improving cognitive functioning and quality of life in older adults. It is hoped that the findings from this study will add to a rapidly growing body of evidence which suggests that these types of interventions may be important for older adults, especially in a society that is facing the economic and social ramifications of an aging population.

\section{Abbreviations}

GP: general practitioner; TICS: Telephone Interview of Cognitive Status; HRR: Heart rate reserve; ACSM: American College of Sports Medicine; RPE: Rate of Perceived Exertion; CLVT: California Verbal Learning Test; COAST: California Older Adults Stroop Test; D-KEFS: Delis-Kaplan Executive Function Scale; WMS: Wechsler Memory Scale; WAIS: Wechsler Adult Intelligence Scale; SDMT: Symbol Digit Modalities Test; TIADL: Timed Instrumental Activities of Daily Living; cm: centimeters; kg: kilograms; mm Hg: millimeters of mercury; bpm: beats per minute; SMWT: Six Minute Walk Test; kp: kiloponds; MAC-Q: Memory Complaint Questionnaire; SF-36: Medical Outcomes Study Short Form; GADS: Geriatric Anxiety and Depression Scale; HRHS: Health-related Hardiness Scale

\section{Competing interests}

The author(s) declare that they have no competing interests.

\section{Authors' contributions}

SO developed the idea for the study. SO, WB, NP and NB were involved in further developing the protocol. SO was responsible for drafting the manuscript and will implement the protocol and collect the data. All authors contributed to the final manuscript. All authors have read and approved the final manuscript.

\section{Acknowledgements}

Kelly Harriden (School of Psychology, Psychiatry and Psychological Medicine: Monash University) assisted with the development of the cognitive training. Associate Professor Ken McFarland (School of Psychology, University of Queensland) developed the Reaction Time tasks.

\section{References}

I. Schaie KW: The course of adult intellectual development. American Psychologist 1994, 49:304-313.

2. Hultsch DF, Hertzog C, Small BJ, McDonald-Miszczak L, Dixon RA: Short-term longitudinal change in cognitive performance in later life. Psychology and Aging 1992, 7:571-584.

3. Cullum S, Huppert FA, McGee M, Dening T, Ahmed A, Paykel ES, Brayne C: Decline across different domains of cognitive function in normal ageing: Results of a longitudinal populationbased study using CAMCOG. International Journal of Geriatric Psychiatry 2000, I 5:853-862.

4. Singer T, Verghaeghen P, Ghisletta P, Lindenberger U, Baltes PB: The fate of cognition in very old age: Six-year longitudinal findings in the Berlin Aging Study (BASE). Psychology and Aging 2003, 18:318-331.

5. Rabbitt PMA, Mclnnes L, Diggle P, Holland F, Bent N, Abson V, Pendleton N, Horan M: The University of Manchester Longitudinal Study of cognition in normal healthy old age, 1983 through 2003. Aging, Neuropsychology and Cognition 2004, I I :245-279.

6. Cahn-Weiner DA, Boyle PA, Malloy PF: Tests of executive function predict instrumental activities of daily living in community-dwelling older individuals. Applied Neuropsychology 2002, 9:|87-|9|.

7. Zimmerman T, McDougall GJ, Becker $\mathrm{H}$ : Older women's cognitive and affective response to moderate drinking. International Journal of Geriatric Psychiatry 2004, I 9: 1095- I 102. 
8. Jonsson L, Lindgren P, Wimo A, Jonsson B, Winblad B: Costs of Mini Mental State Examination-related cognitive impairment. Pharmacoeconomics 1999, 16:409-416.

9. Pedersen BK, Saltin B: Evidence for prescribing exercise as therapy in chronic disease. Scandinavian Journal of Medicine \& Science in Sports 2006, 16:3-63.

10. Colcombe SJ, Kramer AF, McAuley E, Erickson KI, Scalf P: Neurocognitive aging and cardiovascular fitness: Recent findings and future directions. Journal of Molecular Neuroscience 2004, 24:9-14.

II. Dustman RE, Emmerson R, Shearer D: Physical activity, age, and cognitive-neuropsychological function. Journal of Aging and Physical Activity 1994, 2:143-181.

12. Clarkson-Smith L, Hartley AA: Relationships between physical exercise and cognitive abilities in older adults. Psychology and Aging 1989, 4:183-189.

13. Roth DL, Goode KT, Clay OJ, Ball KK: Association of physical activity and visual attention in older adults. Journal of Aging and Health 2003, 15:534-547.

14. Yaffe K, Barnes D, Nevitt M, Lui LY, Covinsky K: A prospective study of physical activity and cognitive decline in elderly women: Women who walk. Archives of Internal Medicine 2001 , | 6 1:1703-1708.

I5. Weuve J, Kang JH, Manson JE, Breteler MMB, Ware JH, Grodstein F: Physical activity, including walking, and cognitive function in older women. JAMA 2004, 292: |454-|46|.

16. Pignatti F, Rozzini R, Trabucchi M: Physical activity and cognitive decline in elderly persons. Archives of Internal Medicine 2002 I 62:361-362.

17. Barnes D, Yaffe K, Satariano WA, Tager IB: A longitudinal study of cardiorespiratory fitness and cognitive function in healthy older adults. JAGS 2003, 5 I:459-465.

18. van Gelder BM, Tijhuis MAR, Kalmijn S, Giampaoli S, Nissinen A, Kromhout D: Physical activity in relation to cognitive decline in elderly men: The FINE Study. Neurology 2004, 63:23 I 6-232I.

19. Dustman RE, Ruhling RO, Russell EM, Shearer DE, Bonekat HW, Shigeoka JW, Wood JS, Bradford DC: Aerobic exercise training and improved neuropsychological function of older individuals. Neurobiology of Aging 1984, 5:35-42.

20. Moul JL, Goldman B, Warren B: Physical activity and cognitive performance in the older population. Journal of Aging and Physical Activity 1995, 3: |35-145.

21. Kramer AF, Hahn S, Cohen NJ, Banich MT, McAuley E, Harrison CR, Chason J, Vakil E, Bardell L, Boileau RA, Colcombe A: Ageing, fitness and neurocognitive function. Nature 1999, 400:418-419.

22. Blumenthal JA, Emery CF, Madden DJ, Schniebolk S, Walsh-Riddle M, George LK, McKee DC, Higginbotham MB, Cobb FR, Coleman RE: Long-term effects of exercise on psychological functioning in older men and women. Journal of Gerontology I991, 46:352-36I.

23. Blumenthal JA, Madden DJ: Effects of aerobic exercise training, age, and physical fitness on memory-search performance. Psychology and Aging 1988, 3:280-285.

24. Hill RD, Storandt M, Malley M: The impact of long-term exercise training on psychological function in older adults. Journals of Gerontology: Psychological Sciences 1993, 48:PI2-PI7.

25. Colcombe S, Kramer AF: Fitness effects on the cognitive function of older adults: A meta-analytic study. Psychological Science 2003, I 4: I 25-130.

26. Colcombe SJ, Erickson KI, Raz N, Webb AG, Cohen NJ, McAuley E, Kramer AF: Aerobic fitness reduces brain tissue loss in aging humans. Journal of Gerontology: Medical Sciences 2003, 58A: I 76- I80.

27. Rogers RL, Meyer JS, Mortel KF: After reaching retirement age physical activity sustains cerebral perfusion and cognition. JAGS 1990, 38:123-128.

28. Poon LW, Chodzko-Zajko W, Tomporowski PD: Active Living, Cognitive Functioning, and Aging. In Aging, Exercise and Cognition Series Volume I. Edited by: Poon LW, Spirduso WW and ChodzkoZajko W. Champaign, IL, Human Kinetics; 2006.

29. Madden DJ, Blumenthal JA, Allen PA, Emery CF: Improving aerobic capacity in healthy older adults does not necessarily lead to improved cognitive performance. Psychology and Aging 1989, 4:307-320.

30. Spirduso WW: Issues of aging, physical activity, cognition, and putative mechanisms for a relationship: A discussion. In Active Living, Cognitive Functioning, and Aging Volume I. Edited by: Poon LW,
Chodzko-Zajko W and Tomporowski PD. Champaign, IL, Human Kinetics; 2006:187-195.

31. Dunn AL, Trivedi MH, Kampert JB, Clark CG, Chambliss HO: Exercise treatment for depression: Efficacy and dose response. Am J Prev Med 2005, 28: I-8.

32. Yaffe K, Blackwell T, Gore R, Sands L, Reus V, Browner WS: Depressive symptoms and cognitive decline in nondemented elderly women: A prospective study. Archives of General Psychiatry 1999, 56:425.

33. Lee C, Russell A: Effects of physical activity on emotional wellbeing among older Australian women: Cross-sectional and longitudinal analyses. Journal of Psychosomatic Research 2003, 54: $155-160$

34. Mohs RC, Ashman TA, Jantzen K, Albert M, Brandt J, Gordon B, Rasmusson X, Grossman M, Jacobs D, Stern Y: A study of the efficacy of a comprehensive memory enhancement program in healthy elderly persons. Psychiatry Research 1998, 77:183-195.

35. Derwinger A, Neely AS, Backman L: Design your own memory strategies! Self-generated strategy training versus mnemonic training in old age: An 8-month follow-up. Neuropsychological Rehabilitation 2005, I 5:37-54.

36. Fabre C, Chamari K, Mucci P, Masse-Biron J, Prefaut C: Improvement of cognitive function by mental and/or individualized aerobic training in healthy elderly subjects. International Journal of Sports Medicine 2002, 23:4 I5-42I.

37. Emery CF, Gatz M: Psychological and cognitive effects of an exercise program for community-residing older adults. Gerontologist 1990, 30: 184-188.

38. Williams P, Lord SR: Effects of group exercise on cognitive functioning and mood in older women. Australian and New Zealand Journal of Public Health 1997, 2 I:45-52.

39. Ball K, Berch DB, Helmers KF, Jobe JB, Leveck MD, Marsiske M, Morris JN, Rebok GW, Smith DM, Tennstedt SL, Unverzagt FW, Willis SL: Effects of cognitive training interventions with older adults: A randomized controlled trial. JAMA 2002, 288:227I-228I.

40. Kramer AF, Willis SL: Enhancing the cognitive vitality of older adults. Current Directions in Psychological Science 2002, I I:I73-I77.

41. Thompson G, Foth D: Cognitive-training programs for older adults: What are they and can they enhance mental fitness? Educational Gerontology 2005, 3 I:603-626.

42. Hultsch DF, Hammer M, Small BJ: Age differences in cognitive performance in later life: Relationships to self-reported health and activity life style. Journal of Gerontology: Psychological Sciences 1993, 48: I-II

43. Hultsch DF, Hertzog C, Small BJ, Dixon RA: Use it or lose it: Engaged lifestyle as a buffer of cognitive decline in aging? Psychology and Aging 1999, 14:245-263.

44. Newson RS, Kemps EB: General lifestyle activities as a predictor of current cognition and cognitive change in older adults: A cross-sectional and longitudinal examination. The Journals of Gerontology 2005, 60B: I I3-I 20.

45. Schooler C, Mulatu MS: The reciprocal effects of leisure time activities and intellectual functioning in older people: A longitudinal analysis. Psychology and Aging 200I, 16:466-482.

46. King AC, Rejeski WJ, Buchner DM: Physical activity interventions targeting older adults: $A$ critical review and recommendations. American Journal of Preventive Medicine 1998, I 5:316-332

47. Resnick B, Spellbring AM: Understanding what motivates older adults to exercise. Journal of Gerontological Nursing 2000, 26:34-42.

48. de Jager CA, Budge MM, Clarke R: Utility of TICS-M for the assessment of cognitive function in older adults. International Journal of Geriatric Psychiatry 2003, I 8:3 I 8-324.

49. Hassmen P, Ceci R, Backman L: Exercise for older women:A training method and its influences on physical and cognitive performance. European Journal of Applied Physiology 1992, 64:460-466.

50. Beller EM, Gebski V, Keech AC: Randomisation in clinical trials. Medical Journal of Australia 2002, I 77:565 -5567.

51. ACSM: ACMS's guidelines for exercise testing and prescription. 7th edition. Baltimore, Lippincott, Williams \& Wilkins; 2005.

52. Borg G: Borg's Perceived Exertion and Pain Scales. Champaign, IL, Human Kinetics; 1998.

53. Welsh KA, Breitner JCS, Magruder-Habib KM: Detection of dementia in the elderly using telephone screening of cogni- 
tive status. Neuropsychiatry, Neuropsychology, and Behavioral Neurology 1993, 6:103-110.

54. Moylan T, Das K, Gibb A, Hill A, Kane A, Lee C, Toye D, Wolstencroft K, Fail M, Stott DJ: Assessment of cognitive function in older hospital inpatients: Is the Telephone Interview for Cognitive Status (TICS-M) a useful alternative to the Mini Mental State Examination? International Journal of Geriatric Psychiatry 2004, 19:1008-1009.

55. Barber M, Stott DJ: Validity of the Telephone Interview for Cognitive Status (TICS) in post-stroke subjects. International Journal of Geriatric Psychiatry 2004, 19:75-79.

56. Delis DC, Kramer JH, Kaplan E, Ober BA: California Verbal Learning Test - Second Edition (CVLT-II). San Antonio, Texas, Psychological Corporation; 2000.

57. Lezak MD, Howieson DB, Loring DW: Neuropsychological Assessment. 4th Edition edition. Oxford, Oxford University Press; 2004.

58. Wechsler D: Wechsler Memory Scale. Third edition manual. San Antonio, The Psychological Corporation; 1997.

59. Pachana N, Thompson LW, Marcopulous BA, Yoash-Gantz R: California Older Adult Stroop Test (COAST): Development of a stroop test adapted for geriatric populations. Clinical Gerontologist 2004, 27:3-22.

60. Delis DC, Kaplan E, Kramer JH: Delis-Kaplan Executive Function System. San Antonio, Psychological Corporation; 2001.

6I. Wechsler D: Wechsler Adult Intelligence Scale-III. San Antonio, The Psychological Corporation; 1997.

62. Smith A: Symbol Digit Modalities Test (SDMT). Manual (revised). Los Angeles, Western Psychological Services; 1982.

63. Owsley C, Sloane M, McGwin GJ, Ball K: Timed instrumental activities of daily living tasks: Relationship to cognitive function and everyday performance assessments in older adults. Gerontology 2002, 48:254-265.

64. Spreen O, Strauss E: A compendium of neuropsychological tests: Administration, norms, and commentary. 2nd edition. New York, Oxford University Press; 1998.

65. Rikli RE, Jones CJ: Senior fitness test. Champaign, IL, Human Kinetics; 1997.

66. Crook TH, Feher EP, Larrabee GJ: Assessment of memory complaint in age-associated memory impairment: The MAC-Q. International Psychogeriatrics 1992, 4:165-I76.

67. McHorney CA, Ware JE, Raczek AE: The MOS 36-item shortform health survey (SF-36): II. Psychometric and clinica tests of validity in measuring physical and mental health constructs. Medical Care 1993, 31:247-263.

68. Ware JE, Snow K, Kosinski M, Gandek B: SF-36 Health Survey: Manual and interpretation guide. Lincoln, RI, Quality Metric Incorporated; 2000.

69. Goldberg D, Bridges K, Duncan-Jones P, Grayson D: Detecting anxiety and depression in general medical settings. British Medical Journal 1988, 297:897-899.

70. Pollock SE, Duffy ME: The Health-Related Hardiness Scale: Development and psychometric analysis. Nursing Research 1990, 39:218-222

7I. MacDonald SWS, Hultsch DF, Dixon RA: Performance variability is related to change in cognition: Evidence from the Victoria Longitudinal Study. Psychology and Aging 2003, 18:510-523.

72. Jacobson NS, Follette WC, Revenstorf D: Psychotherapy outcome research: Methods for reporting variability and evaluating clinical significance. Behavior Therapy 1984, I 5:336-352.

73. Jacobson NS, Truax P: Clinical significance: A statistica approach to defining meaningful change in psychotherapy research. Journal of Consulting and Clinical Psychology I991, 59:12-19.

74. Bortz WM: Foreword. In Active Living, Cognitive Functioning, and Aging Volume I. Edited by: Poon LW, Chodzko-Zajko W and Tomporowski PD. Champaign, IL, Human Kinetics; 2006:vii-viii.

\section{Pre-publication history}

The pre-publication history for this paper can be accessed here:

http://www.biomedcentral.com/1471-2318/7/23/prepub
Publish with Biomed Central and every scientist can read your work free of charge

"BioMed Central will be the most significant development for disseminating the results of biomedical research in our lifetime. " Sir Paul Nurse, Cancer Research UK

Your research papers will be:

- available free of charge to the entire biomedical community

- peer reviewed and published immediately upon acceptance

- cited in PubMed and archived on PubMed Central

- yours - you keep the copyright
BioMedcentral 